J.D. Badia, L. Santonja-Blasco, A. Martínez-Felipe,A. Ribes-Greus. Reprocessed polylactide: studies of thermooxidative decomposition. Bioresource Technology 2012; 114:622-628.

\title{
REPROCESSED POLYLACTIDE: STUDIES OF THERMO-OXIDATIVE DECOMPOSITION
}

\section{J. D. Badia, L. Santonja-Blasco, A. Martínez-Felipe, $\underline{\text { A. Ribes-Greus }}$}

This is an open-access version, according to http://www.sherpa.ac.uk/romeo/issn/0960-8524/es/

Full text available at http://www.sciencedirect.com/science/article/pii/S0960852412003793

DOI: dx.doi.org/10.1016/j.biortech.2012.02.128

Please, cite it as:

J.D. Badia, L. Santonja-Blasco, A. Martínez-Felipe,A. Ribes-Greus. Reprocessed polylactide: studies of thermo-oxidative decomposition. Bioresource Technology 2012; 114:622-628.

Instituto de Tecnología de los Materiales (ITM),

Universidad Politècnica de València (UPV)

Camino de Vera S/N, 46022 Valencia, Spain.

*Corresponding author: A. Ribes-Greus aribes@ter.upv.es 
J.D. Badia, L. Santonja-Blasco, A. Martínez-Felipe,A. Ribes-Greus. Reprocessed polylactide: studies of thermooxidative decomposition. Bioresource Technology 2012; 114:622-628.

\title{
REPROCESSED POLYLACTIDE: STUDIES OF THERMO-OXIDATIVE DECOMPOSITION
}

\author{
J.D. Badia, L. Santonja-Blasco, A. Martínez-Felipe,A. Ribes-Greus* \\ Instituto Tecnológico de Materiales. Universidad Politécnica de Valencia. \\ Camino de Vera, s/n, 46022 Valencia (Spain) \\ *corresponding author: aribes@ter.upv.es
}

Keywords: energetic valorization, polylactide (PLA), thermal stability, thermooxidative decomposition kinetics, evolved-gases analysis (EGA)

\begin{abstract}
:
The combustion process of virgin and reprocessed polylactide (PLA) was simulated by multi-rate linear non-isothermal thermogravimetric experiments under $\mathrm{O}_{2}$.A complete methodology that accounted on the thermal stability and emission of gases was thoroughly developed. A new model, Thermal Decomposition Behavior, and novel parameters, the Zero-Decomposition Temperatures, were used to test the thermal stability of the materials under any linear heating rate. The release of gases was monitored by Evolved Gas Analysis with in-line FT-IR analysis. In addition, a kinetic analysis methodology that accounted for variable activation parameters showed that the decomposition process could be driven by the formation of bubbles in the melt. It was found that the combustion technologies for virgin PLA could be transferred for the energetic valorizationof its recyclates. Combustion was pointed out as appropriate for the energetic valorization of PLA submitted to more than three successive reprocessing cycles.
\end{abstract}


J.D. Badia, L. Santonja-Blasco, A. Martínez-Felipe,A. Ribes-Greus. Reprocessed polylactide: studies of thermooxidative decomposition. Bioresource Technology 2012; 114:622-628.

\section{List of abbreviations}

$\alpha$ : degree of conversion

A:Pre-exponential factor

AIC: Advanced Isoconversional Method

$\beta$ : TGA heating rate

DTG: First-derivative thermogravimetric curve

Ea: Activation energy

$\mathrm{Ea} \alpha:$ Apparent Ea at a fixed $\alpha$

EGA: Evolved Gas Analysis

$f(\alpha)$ : kinetic function

FT-IR: Fourier-Transform InfraRed Spectroscopy

FWO: Flynn-Wall-Ozawa

KAS: Kissinger-Akashira-Sunose

MP: Master-Plots

$n$ : order in kinetic functions $f(\alpha)$

$\mathrm{n}_{\beta}: \mathrm{n}$ averaged from experiments at different $\beta$

n': $\mathrm{n}$ averaged from $\mathrm{n} \beta$

n': $n$ averaged from the n obtained analytically - Eq. (4) -

p: power 
J.D. Badia, L. Santonja-Blasco, A. Martínez-Felipe,A. Ribes-Greus. Reprocessed polylactide: studies of thermooxidative decomposition. Bioresource Technology 2012; 114:622-628.

RPLA-i: reprocessed polylactide

TDB: Thermal Decomposition Behavior

TG: Thermogravimetric curve

TGA: Thermogravimetric analysis

VPLA: Virgin polylactide

ZDT: Zero-Decomposition Temperature 
J.D. Badia, L. Santonja-Blasco, A. Martínez-Felipe,A. Ribes-Greus. Reprocessed polylactide: studies of thermooxidative decomposition. Bioresource Technology 2012; 114:622-628.

\section{Introduction}

The research in the packaging industry within a framework of sustainable development is focused on the use of bio-based materials which accomplish the benefit of coming from renewable resources, and being biodegradable once discarded, within a rational time. In this sense, polylactide (PLA) is an aliphatic polyester that can be obtained from agricultural resources, and reintroduced into the carbon cycle after being used.The increasing know-how in the technology of production of PLA (Gupta and Kumar, 2007) enhances its performance as suitable candidate for replacing commodities at the packaging sector.

Despite the potential of PLA for being treated by biological valorization in composting facilities, the foreseeable high amount of plastic waste invites to diversify and combine the available technologies of valorization. Among current procedures, material valorization by mechanical recycling is widely established (Vilaplana and Karlsson, 2008). It mainly consists in recovery, washing, drying, shredding and processing by means of extrusion or injection.The inherent thermo-mechanical degradation may modify the polymeric structure (Badia et al, 2009) and consequently affect the thermal, rheological and mechanical properties (Strömberg and Karlsson, 2009) (Badia et al, 2012a) thus reducing the performance rates of recycled goods.

A viable solution to manage bio-based recycled plastics waste, when no better performance can be guaranteed, could be the application of thermally-induced valorization technologies, such as pyrolysis and combustion (Al-Salem et al, 2009).

The application of these thermal operations must be carefully handled by technologists during the design of energetic valorization facilities, taking into account 
J.D. Badia, L. Santonja-Blasco, A. Martínez-Felipe,A. Ribes-Greus. Reprocessed polylactide: studies of thermooxidative decomposition. Bioresource Technology 2012; 114:622-628.

the knowledge of the thermal stability and the detection of emitted gases. As well, the characterization of the decomposition kinetics should be considered.

Thermogravimetric analysis (TGA) is a widely used technique to assess the thermal stability and reaction kinetics of biomass (Barnetto et al, 2010) and bio-based polymers (Carrasco et al, 2010). On the other hand, detection techniques such as Fourier Transform -InfraRed Spectroscopy (FT-IR) are widely used hyphenated to TGA for gas detection (Materazzi and Vecchio, 2010).

The majority of current studies on the combustion behavior of PLA are reported from the point of view of the flame retardancy (Bourbigot and Fontaine, 2010), but not approached from the point of view of the management of their wastes. The aim of this work was thus to assess the behavior of mechanically-recycled polylactide submitted to a combustion process, with the purpose of assuring the performance of energetic valorization processes as a contribution for further plastic waste management solutions.

\section{Experimental procedure}

\subsection{Reprocessing simulation and sample preparation}

Polylactide (PLA) 2002D is a thermo-forming grade PLA obtained from Natureworks LLC (Minnetonka, MN) in the form of pellets. Prior to processing, virgin PLA (VPLA) pellets were dried during $2 \mathrm{~h}$ at $80^{\circ} \mathrm{C}$ in a dehumidifier Conair Micro-D FCO 1500/3 (UK), in order to remove as much humidity as possible from PLA flakes.Afterwards, samples were processed by means of injection molding with an Arburg420 C 1000-350 (Germany) injector, single-screw model (diameter $\Phi=35 \mathrm{~mm}$, length $/ \Phi=23)$. Successive processing steps were applied under the same conditions (temperature gradient set from hopper to die: 160, 170, 190, 200 and $190^{\circ} \mathrm{C}$; moulds set at $15^{\circ} \mathrm{C}$; cooling time residence $\sim 40 \mathrm{~s}$ and total residence time $\left.\sim 60 \mathrm{~s}\right)$. Samples were 
J.D. Badia, L. Santonja-Blasco, A. Martínez-Felipe,A. Ribes-Greus. Reprocessed polylactide: studies of thermooxidative decomposition. Bioresource Technology 2012; 114:622-628.

dried before each processing cycle. After injection, a fraction of the samples were kept as test specimens and the rest was ground by means of a cutting mill Retsch SM2000 (UK), which provide pellets of size $\mathrm{d}<20 \mathrm{~mm}$ to be fed back into the recirculation process. Up to five processing cycles were applied to obtain the different testing specimens of reprocessed PLA (RPLA-i, with i: 1-5).

\subsection{Thermogravimetric experiments}

Multi-rate linear non-isothermal thermogravimetric experiments were carried out in a Mettler-Toledo TGA/SDTA 851 (Columbus, OH). Samples weighing 5 mg were heated in an alumina holder with capacity for $70 \mu \mathrm{L}$. Experiments were performed from 25 to $750{ }^{\circ} \mathrm{C}$ at different heating rates $\left(\beta=2,5,7,10,12,15^{\circ} \mathrm{C} \cdot \mathrm{min}^{-1}\right)$, under constant flow of $50 \mathrm{~mL} \cdot \mathrm{min}^{-1}$ of oxygen. Experiments were repeated at least three times, and the averages were considered as representative values. The assessment was performed with the help of the software STAR ${ }^{\mathrm{e}} 9.10$ from Mettler-Toledo.

Results are plotted in terms of $\left\{\right.$ average, $\left.d_{\text {evmax }}, d_{e v \min }\right\}$, where devmax $=\max$ (data)-average (data), and devmin $=$ average (data) $-\min$ (data). Tabulated errors were obtained by dividing the standard deviation by the average of data.

\subsection{Evolved Gas Analysis.}

Evolved Gas Analysis (EGA) was applied to fumes released by combustion by means of coupled TGA/FT-IR. In this case, the TGA analysis was focused on a temperature range in which the main decomposition range of PLA occurred, from 180 to $500{ }^{\circ} \mathrm{C}$, by means of a heating rate of $1{ }^{\circ} \mathrm{C} \cdot \mathrm{min}^{-1}$. Samples weighing $\sim 40 \mathrm{mg}$ were heated in an alumina holder with capacity for $900 \mu \mathrm{L}$. The flow rate of the oxygen was set to $25 \mathrm{~mL} \cdot \mathrm{min}^{-1}$, according to technical specifications. FT-IR gas-phase spectra were collected by a previously calibrated Thermo Nicolet 5700 FT-IR Spectrometer (MA, 
J.D. Badia, L. Santonja-Blasco, A. Martínez-Felipe,A. Ribes-Greus. Reprocessed polylactide: studies of thermooxidative decomposition. Bioresource Technology 2012; 114:622-628.

USA), from 4000 to $600 \mathrm{~cm}^{-1}$ of wavenumber, at a resolution of $4 \mathrm{~cm}^{-1}$. Both transfer line and gas cell were kept at $250{ }^{\circ} \mathrm{C}$ to prevent gas condensation. 16 co-added spectra were recorded every $30 \mathrm{~s}$ to assure accuracy of the temperature scanning. The GramSchmidt plots as well as its corresponding 3D FT-IR spectra were analyzed with the help of software OMNIC 7.0.2D-IR correlation spectroscopy was performed by means of the software 2Dshige (Morita, 2005).

\section{Results and discussion}

The potential of the use multi-rate thermogravimetric analyses, coupled to FT-IR, to approach the behavior of PLA and its subsequent recyclates under a thermo-oxidative decomposition process is discussed in detail in this section. The assessment comprises (i) the description of the decomposition profiles; (ii) the use of a novel model to functionalize the thermal stability at different heating rates; (iii) the application of a thorough kinetic strategy pursued for ascertaining the kinetics of decomposition accounting for variable activation parameters; and (iv) the detection of gases evolved during the decomposition.

\subsection{Description of the thermo-oxidative decomposition profiles}

Fig 1 a shows the thermogravimetric curve at the heating rate $\beta=5^{\circ} \mathrm{C} \cdot \mathrm{min}^{-1}$ of virgin and fifth reprocessed PLAs. The rest of reprocessed materials showed intermediate profiles. As expected, higher $\beta$ led to shift the thermograms towards higher temperatures, but the graphs have not been shown for the sake of clarity.The thermooxidative decomposition took place through a two-stage profile. The first mass-loss took place from $250{ }^{\circ} \mathrm{C}$ to $370{ }^{\circ} \mathrm{C}$, consuming nearly $96-98 \%$ of the material, which can be addressed to the decomposition of the backbone. Immediately afterwards, the second step appeared from $370{ }^{\circ} \mathrm{C}$ to $400{ }^{\circ} \mathrm{C}$, eliminating $4-2 \%$ of the polymer, without 
J.D. Badia, L. Santonja-Blasco, A. Martínez-Felipe,A. Ribes-Greus. Reprocessed polylactide: studies of thermooxidative decomposition. Bioresource Technology 2012; 114:622-628.

presence of char, as can be seen in the inset of Fig 1a. As expected, lower temperatures than those used in inert conditions (Badia et al, 2012c) were necessary under an oxidizing environment. For further calculations, the second step under $\mathrm{O}_{2}$ was considered negligible if compared with the main decomposition and therefore the study was focused on the first mass-loss region.

A complete description of the thermo-oxidative decomposition of PLA recyclates is given in terms of thermal stability, decomposition kinetics, and detection of evolved gases, as a first approach to be used in combustion facilities.
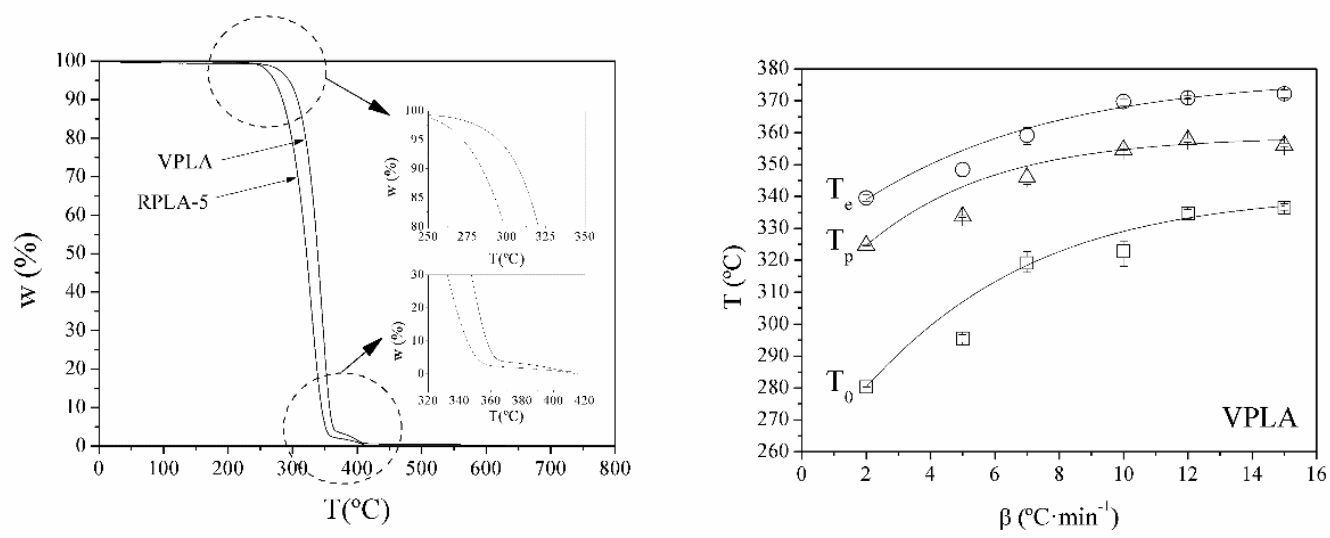

Figure 1. (a) Mass-loss profiles of virgin PLA and PLA reprocessed five times. Inset: detail of onset and endset of decomposition. (b) Application of the TDB model to fit the evolution of the characteristic thermogravimetric temperatures $(0$ : onset, p: peak, e: endset), for the case of virgin PLA.

\subsection{On the use of the TDB model to describe the thermal stability of PLA under any}

linear heating rate

With the aim of assessing the thermal stabilityof PLA and its further recyclates, the corresponding decomposition onset $T_{0}$ and endset $T_{e}$ temperatures were obtained by a tangential intercept method onto the thermogravimetric curves for the whole process. 
J.D. Badia, L. Santonja-Blasco, A. Martínez-Felipe,A. Ribes-Greus. Reprocessed polylactide: studies of thermooxidative decomposition. Bioresource Technology 2012; 114:622-628.

Likewise, the temperature at the maximum decomposition rate, i.e. the peak temperature $T_{p}$ of the differential thermogravimetric curve was also considered for all applied heating rates $\beta$. To help enhance the analysis of the influence of the $\beta$ on the characteristic decomposition temperatures and functionalize the thermal stability of PLA under combustion processes, the so-called Thermal Decomposition Behavior TDB model given in Eq. (1) was proposed. An example of its validity ${ }^{1}$ is shown in Fig. 1b for the case of VPLA.

$$
\operatorname{TDB}(\beta)=a \cdot\left(1+b \cdot e^{-k \cdot \beta}\right)^{-1}(1)
$$

In order to evaluate the differences in thermal stability under combustion conditions due to reprocessing, instead of choosing the experimental temperatures obtained at a specific $\beta$, the so-called onset Zero-Decomposition Temperature $Z D T_{0}$ was used, since this parameter is related to the trigger of the decomposition, and was obtained by extrapolating the $T D B$ fitting of $T_{0}$ to infinitely low $\beta$ (Badia et al, 2012c). Table 1 shows the results of the proposed procedure. A sharp increase in $Z D T_{0}$ of $\sim 30$ ${ }^{\circ} \mathrm{C}$ was registered from virgin PLA up to the second recyclate, dropping $\sim 50{ }^{\circ} \mathrm{C}$ when passed the third recyclate. A previous study by means of Differential Scanning Calorimetry (Badia et al, 2012b) showed that the influence of reprocessing on PLA structure formed shorter chains up to the third recyclate. Thus the apparition of new sites liable to $\mathrm{O}_{2}$ in the PLA structure may promote faster decomposition at lower temperatures at high reprocessing cycles, which may need lower temperatures to start their decompositions.

\footnotetext{
${ }^{1}$ See results of fitting in the supplementary material
} 
Table 1 Results of fitting $\mathrm{T} 0=\mathrm{f}(\beta)$ to Eq. (1), along with the onset Zero-Decomposition Temperature obtained by extrapolating Eq (1) to $\beta \rightarrow 0$.

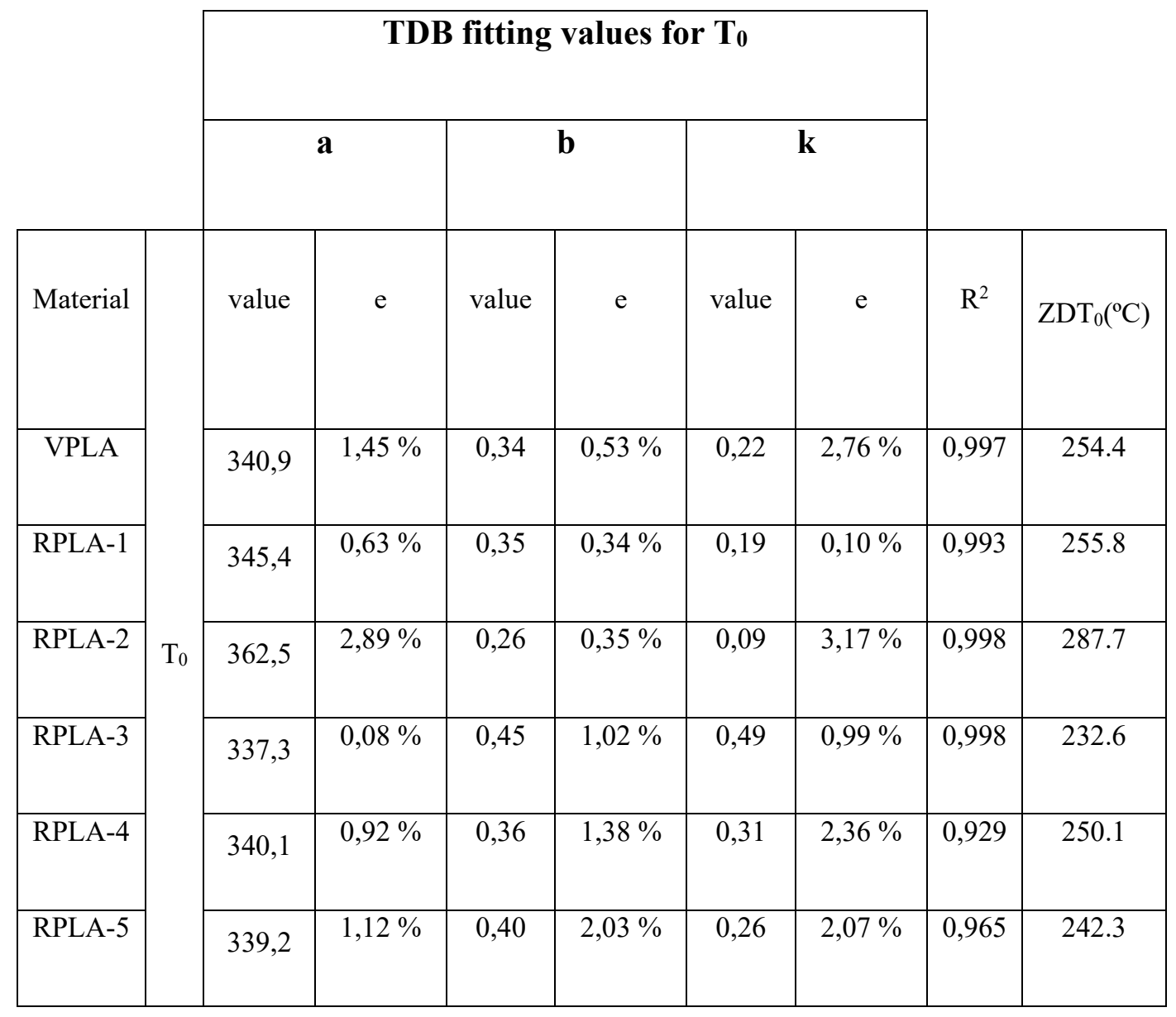

\subsection{Studies on the kinetics of thermo-oxidative decomposition}

The intrinsic kinetics of solid-state decompositions, are usually described by a simplified reaction pathway in terms of three parameters: apparent activation energy $E a$, pre-exponential factor $A$ and kinetic function $f(\alpha)$ with reaction order $n$, conforming the so-called kinetic triplet. In this section, the results of the application of a detailed strategy are shown, according to the following steps: (i)the application of isoconversional methods to assess the evolution of the apparent activation energy, along with the proposal of a novel model to mathematically describe this evolution; (ii) the evaluation of the kinetic model, in terms of variable apparent activation energy and reaction order; (iii) the assessment of the variation of the pre-exponential factor, in order 
J.D. Badia, L. Santonja-Blasco, A. Martínez-Felipe,A. Ribes-Greus. Reprocessed polylactide: studies of thermooxidative decomposition. Bioresource Technology 2012; 114:622-628.

to complete the kinetic triplet. As a result, instead of a kinetic triplet comprised by three constant parameters, this methodology provides the variation of the kinetic triplet along the decomposition process. Finally, the differences between the decomposition of virgin PLA and its recyclates are discussed.

\subsubsection{Evolution of the apparent activation energy}

A significant variation in activation energy $\left(E a_{\alpha}\right)$ along the conversion range $\alpha\left(\alpha=\left(m_{0^{-}}\right.\right.$ $\left.\left.m_{t}\right) /\left(m_{0}-m_{\infty}\right)\right)$, where $m$ was the mass and subscripts $0, t$ and $\infty$ stand for initial, actual and final, respectively), was found, as shown in Figure 2. This variation was detected by the application of three suitable integral isoconversional methods,namelylinear Flynn-Wall-Ozawa (FWO) (Flynn and Wall, 1966) (Ozawa, 1970), linear KissingerAkahira-Sunose $(K A S)$ (Kissinger, 1957) (Akahira and Sunose, 1971), and non-linear Advanced Isoconversional Method (AIC) (Vyazovkin, 1997). As can be seen in Fig 2a, the three methods offered similar $E a_{\alpha}$ values for the assessment of virgin PLA, showing a good coincidence along the decomposition reaction, as also obtained for the rest of reprocessed materials.Fig $2 \mathbf{b}$ and $\mathbf{2 c}$ show the evolution of the $E a_{\alpha}$ averaged from the application of the three isoconversional methods at each $\alpha$, while Table 2 gathers the averaged $E a$ values obtained by each method averaged along the whole $\alpha$ range. The $E a_{\alpha}$ behaved increasing from values $\sim 40-60 \mathrm{~kJ} \cdot \mathrm{mol}^{-1}$ at low conversions $\alpha$ to values $\sim$ $150-170 \mathrm{~kJ} \cdot \mathrm{mol}^{-1}$ at the end of the decomposition. It should be pointed out that the large obtained deviation values ( $€ 18-40 \%)$ did not permit to apply a simplified kinetic triplet (Badia et al, 2012c) that is, with constant activation parameters, and therefore the variation of $E a, A$ and $n$ in $f(\alpha)$ was considered. 
J.D. Badia, L. Santonja-Blasco, A. Martínez-Felipe,A. Ribes-Greus. Reprocessed polylactide: studies of thermooxidative decomposition. Bioresource Technology 2012; 114:622-628.

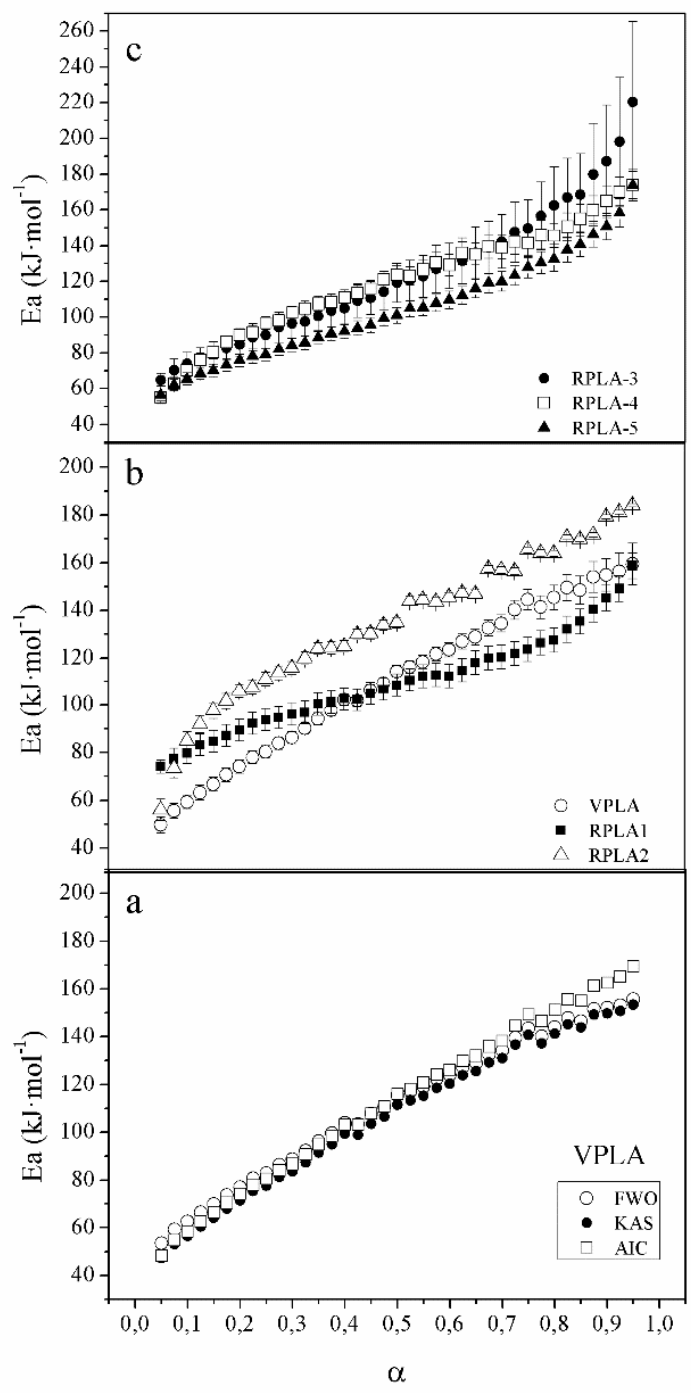

Figure 2.(a) Apparent Ea obtained for VPLA by means of FWO, KAS and AIC methods; (b) Average isoconversional activation energy of VPLA, RPLA-1 and RPLA2; (c) Evolution of average isoconversional activation energy for RPLA-3, RPLA-4 and RPLA-5. 
J.D. Badia, L. Santonja-Blasco, A. Martínez-Felipe,A. Ribes-Greus. Reprocessed polylactide: studies of thermooxidative decomposition. Bioresource Technology 2012; 114:622-628.

Table 2.Average activation energy along the thermo-oxidative decomposition process, as obtained by different isoconversional methods.

\begin{tabular}{|c|c|c|c|c|c|c|}
\hline \multirow[b]{3}{*}{ Material } & \multicolumn{6}{|c|}{ Activation energy averaged from the $\alpha$ range } \\
\hline & \multicolumn{2}{|l|}{ FWo } & \multicolumn{2}{|l|}{ KAS } & \multicolumn{2}{|l|}{ AIC } \\
\hline & $\mathrm{Ea}\left(\mathrm{kJ} \cdot \mathrm{mol}^{-1}\right)$ & e $(\%)$ & $\mathrm{Ea}\left(\mathrm{kJ} \cdot \mathrm{mol}^{-1}\right)$ & e $(\%)$ & $\mathrm{Ea}\left(\mathrm{kJ} \cdot \mathrm{mol}^{-1}\right)$ & $e(\%)$ \\
\hline VPLA & 101 & 30.7 & 96 & 31.2 & 112 & 25.0 \\
\hline RPLA-1 & 106 & 19.8 & 102 & 21.5 & 97 & 23.7 \\
\hline RPLA-2 & 127 & 30.6 & 123 & 33.3 & 120 & 31.0 \\
\hline RPLA-3 & 114 & 29.8 & 116 & 28.4 & 103 & 27.3 \\
\hline RPLA-4 & 121 & 21.8 & 119 & 19.6 & 114 & 18.5 \\
\hline RPLA-5 & 97 & 27.8 & 92 & 30.4 & 89 & 39.3 \\
\hline
\end{tabular}

The physical meaning underlying the evolution of the apparent activation energy has been a matter of profuse debate (Galwey, 2003) (Vyazovkin, 2003). It seems reliable to understand that the increase of $E a_{\alpha}$ is consistent with the decomposition of the residual material which is becoming more refractory as temperature increases (Vyazovkin et al, 2011). In any case, the mathematical characterization of its evolution may help control its kinetics. In order to define the evolution of $E a_{\alpha}$ throughout the thermo-oxidative decomposition process, a powered equation was chosen, based on the Freundlich model (Freundlich, 1928) for growing behaviors, according to Eq. (2):

$$
E a(\alpha)=E a_{o}^{*}+\sum_{i} b_{i} \cdot \alpha^{p_{i}}
$$

,where $i$ was the number of slopes in the curve, $E a^{*}{ }_{0}$ a fictive activation energy at $\alpha=0$, $b$ a fitting parameter and $p$ a power that accounted for the shape of the curve; whether 
J.D. Badia, L. Santonja-Blasco, A. Martínez-Felipe,A. Ribes-Greus. Reprocessed polylactide: studies of thermooxidative decomposition. Bioresource Technology 2012; 114:622-628.

$p<1$, the increase is decelerative, and if $p>1$, the increase is accelerative. Table 3 shows the powers resulted from the fitting of Eq. (2) to the $E a_{\alpha}$ evolution of all materials. Generally, two slopes could be considered, as described by $E a_{\alpha}$ in Figs $2 \mathrm{~b}$ and 2c, and thus $\mathrm{i}=1,2$ in Eq. (2). As can be seen, virgin PLA only showed a decelerative curve, while the recyclates started with a decelerative trend and presented a change in tendency at advanced conversions, rapidly increasing its $E a_{\alpha}$ in an accelerative fashion. Accordingly, it can be also observed how the smaller $p_{1}$ the faster initial increases and vice versa, as clearly happened from VPLA to RPLA-2; on the other hand, the smaller $p_{2}$ the brusquer changes in tendency to faster $E a_{\alpha}$ increases along the reaction, as shown for RPLA-3 to 5. The functionalizing of $E a_{\alpha}$ will be useful for further analysis in which the variation of the activation energy is considered.

Table 3. Results of fitting of Ea and $\ln \mathrm{A}$ to Eq. (2)

\begin{tabular}{|c|c|c|c|c|c|c|c|c|c|c|}
\hline \multirow[b]{3}{*}{ Material } & \multicolumn{5}{|c|}{ ACTIVATION ENERGY / Ea } & \multicolumn{5}{|c|}{ PRE-EXPONENTIAL FACTOR / lnA } \\
\hline & \multicolumn{2}{|l|}{$\mathbf{p}_{1}$} & \multicolumn{2}{|l|}{$\mathbf{p}_{2}$} & \multirow{2}{*}{$R^{2}(\%)$} & \multicolumn{2}{|l|}{$\mathbf{p}_{1}$} & \multicolumn{2}{|l|}{$\mathbf{p}_{2}$} & \multirow{2}{*}{$R^{2}(\%)$} \\
\hline & value & e $(\%)$ & value & e $(\%)$ & & value & $\mathrm{e}(\%)$ & value & e $(\%)$ & \\
\hline VPLA & 0.791 & 2.61 & --- & --- & 99.82 & 0.746 & 2.82 & --- & --- & 99.64 \\
\hline RPLA-1 & 0.591 & 6.39 & 9.352 & 7.81 & 99.85 & 0.672 & 7.99 & 9.467 & 8.30 & 99.80 \\
\hline RPLA-2 & 0.413 & 2.46 & 11.783 & 2.89 & 99.06 & 0.399 & 7.22 & 10.63 & 4.11 & 99.64 \\
\hline RPLA-3 & 0.746 & 6.55 & 5.737 & 8.40 & 99.82 & 0.712 & 7.86 & 5.732 & 8.51 & 99.79 \\
\hline RPLA-4 & 0.357 & 8.40 & 8.294 & 3.96 & 99.81 & 0.342 & 7.08 & 10.47 & 4.23 & 99.68 \\
\hline RPLA-5 & 0.807 & 6.93 & 7.545 & 6.10 & 99.79 & 0.775 & 7.65 & 7.377 & 6.52 & 99.69 \\
\hline
\end{tabular}


J.D. Badia, L. Santonja-Blasco, A. Martínez-Felipe,A. Ribes-Greus. Reprocessed polylactide: studies of thermooxidative decomposition. Bioresource Technology 2012; 114:622-628.

\subsubsection{How to obtain the kinetic model with varying Ea?}

The next step in order to complete the kinetic triplet was to achieve the mathematical model of the decomposition that should best describe the combustion process along the valorization process. Thus, the kinetic function $f(\alpha)$ was approachedby the use of reduced differential Master-Plots $M P f_{e}$ (Gotor et al, 2000). Eq.

(3) was thus used for determining which family of theoretical curves $M P f_{t}$, namely, nucleation and growth $\left(A_{n}\right)$, n-order reaction $\left(F_{n}\right)$, reaction-controlled $\left(R_{n}\right)$ and diffusioncontrolled $\left(D_{n}\right)$-see description elsewhere (Khawan and Flanagan, 2006)- was more suitable to describe the experimental behavior of virgin and reprocessed PLAs facing combustion.

$$
\Phi(f, \alpha)=\sum_{\beta}\left(\sum_{\alpha=0}^{1}\left[M P f_{t}(\alpha)-M P f_{e}(\alpha)\right]^{2}\right) ; \Delta \alpha=0.025 ; \forall f
$$

The minimization of $\Phi$ in Eq. (3) was achieved by the model $A_{n}$, with $n$ ranging from 2 to 4 depending on the heating rate $\beta$ of the analysis, as shown in Table 4. Fig 3 shows the abacus of the theoretical differential master plots in comparison with those experimental for VPLA and RPLA-1, as an example of the accuracy of this methodology to predict the model of decomposition. 
Table 4.n $\beta$ :Values of $\mathrm{n}$ of the Anmodel found for the thermo-oxidative decomposition of PLA and its recyclates at different $\beta ; n$ ': average of $n \beta ; n$ ' : values obtained from the minimization method, using Eq (4).

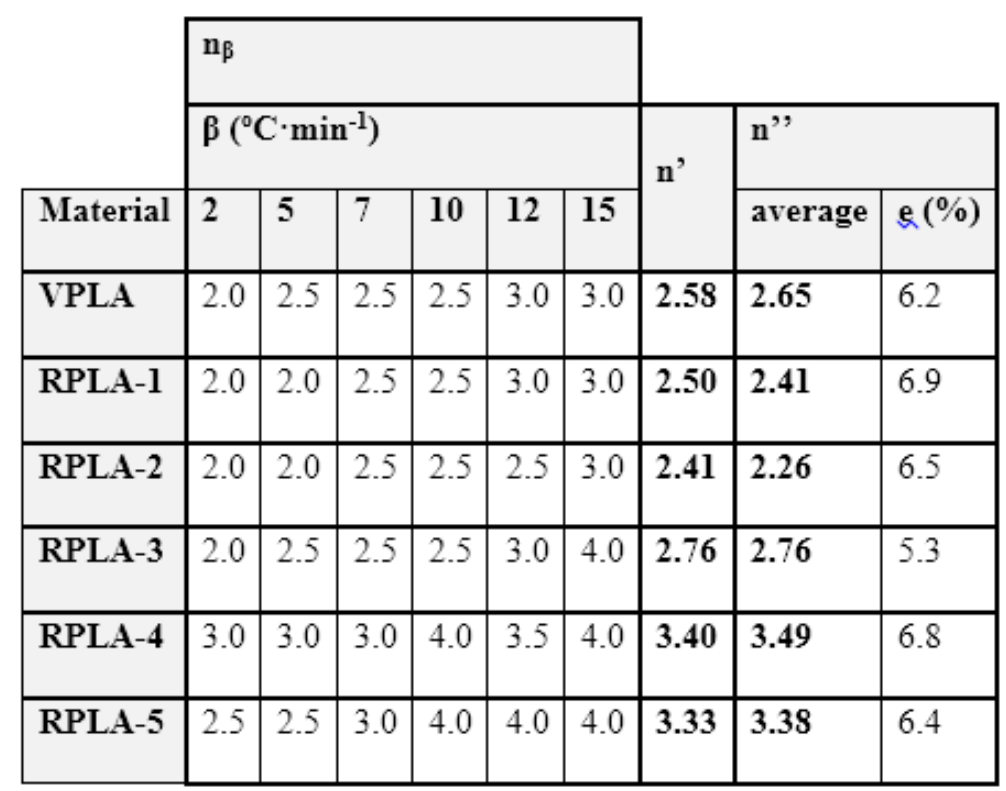

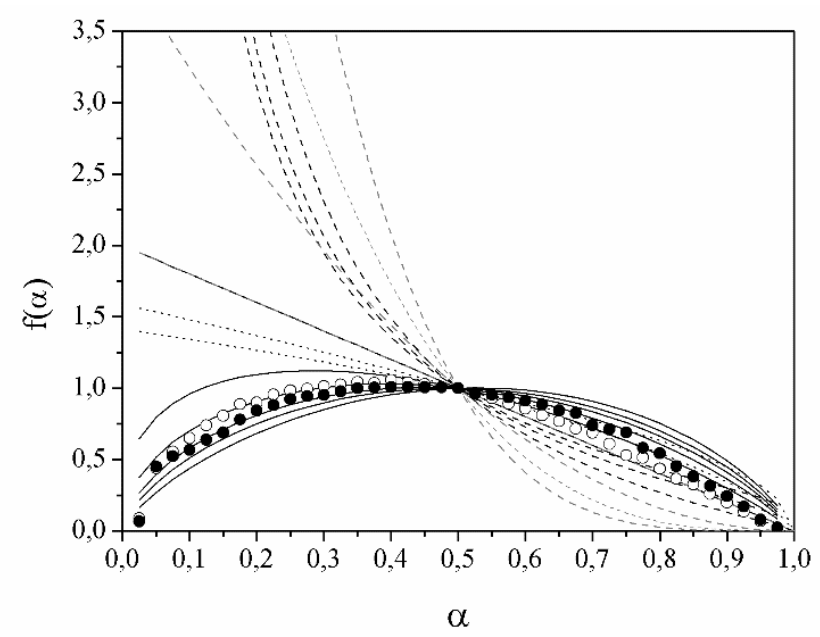

Figure 3.Master plots based on the differential form of the general kinetic law compared to experimental data obtained for thermo-oxidative decomposition of VPLA (hollow circles) and RPLA-1 (full circle). Kinetic models: An (nucleation and growth, solid black lines), Fn (n-order reactions, solid grey lines), Rn (reaction-controlled, pointed lines), Dn (diffusion-controlled, dashed lines) 
The next step consisted in determining the value of $n$ for each conversion degree $\alpha$, which was achieved by the minimization of $\xi$ in Eq. (4), being $i$ the counter of the $h$ experiments carried out at different heating rates $\beta$. This equation compared the $E a_{\alpha}$ obtained by the isoconversional methods to the $E a$ given by the Coats-Redfern (Coats and Redfern, 1964) method in Eq. (5), using the mathematical expression of the kinetic model $A_{n}$, and took into account the Perez-Maqueda et al criterion (Pérez-Maqueda et al, 2002); that is, the independence of the activation parameters on $\beta$. Since the analysis was performed at particular $\alpha$ values, considering all results obtained at all $\beta$, the analysis could be considered also isoconversional.

$$
\xi(n, \alpha)=\sum_{i}^{h}\left|(-R) \cdot \frac{d}{d T}\left(\frac{\ln \left(\beta_{i} \cdot T^{-2}(-\ln (1-\alpha))^{\frac{1}{n}}\right)}{T^{-1}}\right)-E a_{\alpha}\right|
$$

Fig 4 shows the evolution of $n$ along the $\alpha$ range, which could be assumed to be almost constant within a confidence margin lower than 7\%, as shown by $n$ ' ' in Table 3.Therefore, the suitability of the model $A_{n}$ was strengthened. In addition, the possibility of using $n^{\prime}$ (average of $n_{\beta}$, those are, the $n$ individually obtained for each material at each heating rate $\beta$ ) was remarked, since closer values to those given by the analytical procedure ( $n$ ') were obtained, thus permitting to continue the calculations with less time-consuming computations. 


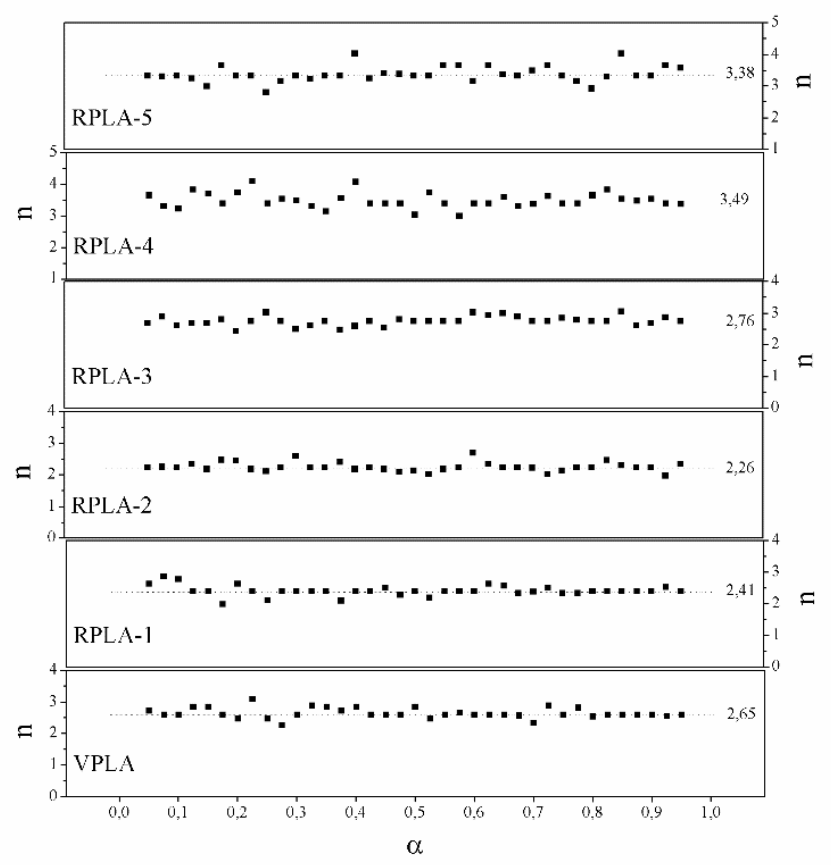

Figure 4. Evolution of $n$ in the $A_{n}$ model of thermo-oxidative decomposition of virgin PLA and its successive recyclates. The number is the average of data (n'' in Table 3)

\subsubsection{Closing the kinetic triplet: pre-exponential factor}

Finally, the evolution of the pre-exponential factor along the $\alpha$ decomposition range $\left(\ln A_{\alpha}\right)$ was also isoconversionally obtained from the intercept at the origin in Eq. (5), considering the data at all heating rates $\beta$.

$$
\left[\ln \frac{\left.\beta \cdot(-\ln (1-\alpha))^{\frac{1}{n}}\right|_{\alpha}}{T^{2}}\right]_{y}=\ln \frac{A_{\alpha} \cdot R}{E a_{\alpha}}+\frac{E a_{\alpha}}{R} \cdot\left[\frac{1}{T}\right]_{x}
$$

Fig5a shows the evolution of $\ln A_{\alpha}$ for VPLA and RPLA-2 as example. The rest are not presented to prevent overlapping curves. It can be seen how $\ln A_{\alpha}$ was strongly connected to the behavior of $E a_{\alpha}$, since both presented similar profiles along the $\alpha$ range. Therefore, one may suggest that the application of Eq. (2) might also be suitable for fitting the experimental data and thus provide a mathematical description of $\ln A_{\alpha}$ evolution. The accuracy of the fitting can be seen at Fig 5a and assessed at Table 2. The powers $p_{1}$ and $p_{2}$ were of the same order than those obtained for $E a_{\alpha}$, thus permitting the 
use of the powers previously obtained for $E a_{\alpha}$ as initialization values in the iteration process of the fitting of $\ln A \alpha$.
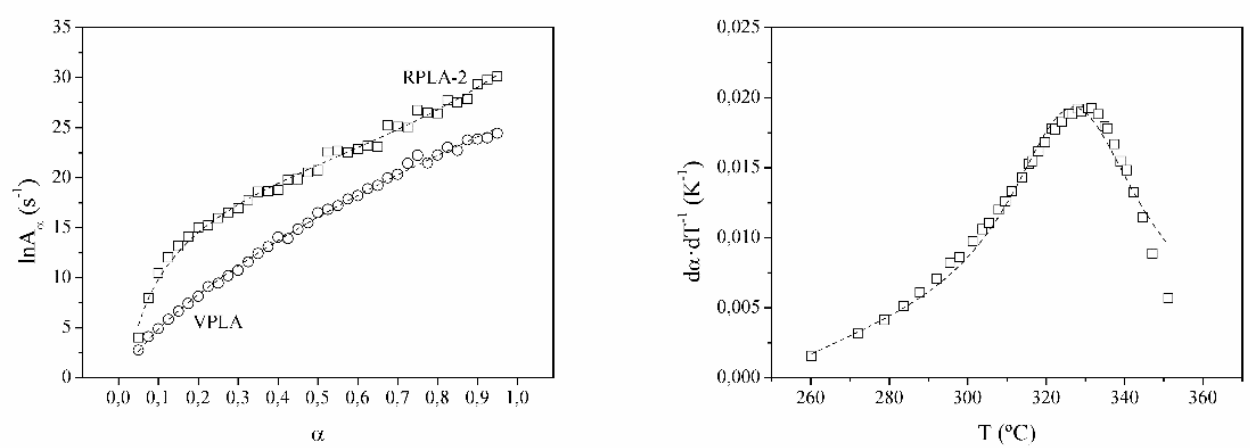

Figure 5. (a)lnA evolution given for VPLA and RPLA-2 as an example of the goodness of fitting of Eq. (2) to explain its behavior along the $\alpha$ range. Hollow symbols: obtained $\ln A \alpha$ values; dashed lines: computed fittings.(b) Comparison of experimental DTG curves (symbols) to computed kinetic functions (dashed lines) obtained from the kinetic methodology.

Finally, the kinetic triplet was completed and the mathematical description of the combustion process of PLA and its recyclates was resolved. Fig $\mathbf{5 b}$ shows for virgin PLA the comparison between the experimental points of the differential thermogravimetric curve in terms of conversion degree, and the mathematical description obtained by the kinetic analysis for variable activation parameters. Similar results were found for the rest of recyclates. The proposed methodology thus stands out as a suitable and effective tool to model the kinetic behavior of virgin and reprocessed PLA under thermally-induced energetic valorizations.

\subsubsection{Effects of reprocessing on the decomposition kinetics of PLA.}

Results concluded that virgin PLA and its recyclates followed an $A_{n}$ kinetic model (growth of previously formed nuclei) during their combustion. This kind of kinetic 
J.D. Badia, L. Santonja-Blasco, A. Martínez-Felipe,A. Ribes-Greus. Reprocessed polylactide: studies of thermooxidative decomposition. Bioresource Technology 2012; 114:622-628.

model is scarcely reported in studies dealingwith thermal decomposition processes of polymers -see discussion elsewhere (Badia et al, 2010)-. However, the model $A_{n}$ indicates the presence of active zones more chemically liable to thermo-oxidative decomposition, which activate the formation and growth of gas bubbles in the polymer melt.

With regards to the evolution of the apparent activation energy, as expected, the $E a_{\alpha}$ needed to trigger the decomposition under oxidative conditions was lower than under inert conditions, i.e. pyrolysis(Badia et al, 2012c), since the reactions were enhanced by oxidation processes which led to the formation of radical species such as carboxylic-ended species that accelerated the decomposition (Bikiaris and Karayannidis, 1999) (Liu et al, 2006). Concerning the differences in $E a_{\alpha}$ among virgin material and recyclates facing the thermo-oxidative decomposition, it was assessed in terms of the apparent activation energy at the initial stages of combustion. For instance, selecting the $E a_{\alpha}$ at a conversion rate of 0.2 (that is, $E a_{0.2}$ ), a similar profile than that shown by the onset Zero-decomposition Temperature $Z D T_{0}$ at the studies of thermal stability was found. An increase up to the second recyclate was registered, which was not followed by the successive reprocessed materials, which kept their Ea0.2 at lower values. Thus the apparition of reaction sites liable to $\mathrm{O}_{2}$ in the PLA structure promoted the decomposition with less demanded energy from the third reprocessing cycle on, also pointing out the feasibility of combustion to valorize highly reprocessed polylactide.

\subsection{Evolved Gases Analysis by in-line FT/IR}

Finally, to exploit the potential of TGA to simulate the thermo-oxidative decomposition of PLA under combustion conditions, the emission of gases was monitored by in-line FT-IR analysis. Main detected evolved species were: carbon 
J.D. Badia, L. Santonja-Blasco, A. Martínez-Felipe,A. Ribes-Greus. Reprocessed polylactide: studies of thermooxidative decomposition. Bioresource Technology 2012; 114:622-628.

dioxide $\left[2349 \mathrm{~cm}^{-1} \mathrm{vas}_{\mathrm{as}}(\mathrm{O}=\mathrm{C}=\mathrm{O})\right]$; carbon monoxide $\left[2174 / 2116 \mathrm{~cm}^{-1}\right.$

$v(\mathrm{C} \equiv \mathrm{O})]$; acetaldehyde $\left[2968 \mathrm{~cm}^{-1} v\left(\mathrm{CH}_{3}\right), 2740 \mathrm{~cm}^{-1} v(\mathrm{CHO}), 1762 \mathrm{~cm}^{-1} v(\mathrm{C}=\mathrm{O})\right.$,

$1414 / 1371 \mathrm{~cm}^{-1} \delta\left(\mathrm{CH}_{3}\right)$ and $\left.1127 \mathrm{~cm}^{-1} v(\mathrm{C}-\mathrm{O})\right]$; acetic acid $\left[3586 \mathrm{~cm}^{-1} \mathrm{v}(\mathrm{H}-\mathrm{O}), 1778 \mathrm{~cm}^{-1}\right.$ $v(\mathrm{C}=\mathrm{O})$ ] which may be formed by oxidation of acetaldehyde;short-chain acids and their dimers and trimers $\left[3589 \mathrm{~cm}^{-1} v(\mathrm{OH}), 2952 \mathrm{~cm}^{-1} v\left(\mathrm{CH}_{2}\right), 2816 \mathrm{~cm}^{-1} v(\mathrm{CH}), 1778 \mathrm{~cm}^{-1}\right.$ $v(C=O)$, and 1164/1107 $\left.\mathrm{cm}^{-1} v(\mathrm{C}-\mathrm{O})\right]$ (Vogel and Siesler, 2008); and traces of lactide[3008 $\mathrm{cm}^{-1} v(\mathrm{CH}), 2952 \mathrm{~cm}^{-1} v_{\text {as }}\left(\mathrm{CH}_{3}\right), 2893 \mathrm{~cm}^{-1} v_{\mathrm{s}}\left(\mathrm{CH}_{3}\right), 1796 \mathrm{~cm}^{-1} v(\mathrm{C}=\mathrm{O}), 1365$ $\mathrm{cm}^{-1} \delta\left(\mathrm{CH}_{3}\right), 1248 / 1108 \mathrm{~cm}^{-1} \mathrm{v}(\mathrm{C}-\mathrm{O}-\mathrm{C})$ and $932 \mathrm{~cm}^{-1}$ corresponding to the ring skeletal vibration] and water [3900-3400 $\left.\mathrm{cm}^{-1} v(\mathrm{H}-\mathrm{O}), 1800-1300 \mathrm{~cm}^{-1} \delta(\mathrm{H}-\mathrm{O})\right]$. The gases were similar to those obtained during the pyrolysis (Badia et al, 2012c). As main differences, carbon mono- and di- oxides evolved with bigger intensity, due to the combustion processes were enhanced; the bands of acetaldehyde decreased, and those related to acetic acid slightly increased, due to the oxidation effect of $\mathrm{O}_{2}$; as well, lactide still appeared, but its bands were overlapped along with those corresponding to short-chain acids and their dimers and trimers and thus a finer identification was complicated. The application of 2D-correlation spectra (Noda, 1990) gave an asynchronous spectrum where positive cross-peaks at $(2378,1796)$ and $(2316,1796)$ and a negative cross-peak at $(2116,1796)$, which exposed that $\mathrm{CO}_{2}$ was evolved before the main decomposition products with $v(\mathrm{C}=\mathrm{O})$ vibrations, while $\mathrm{CO}$ was released afterwards.

After mechanical reprocessing, the chemical nature of polylactide was essentially the same (Badia et al, 2011b), though shorter chains can be obtained (Badia et al, 2012b), and thus no differences were found at the IR spectra of the evolved gases of reprocessed PLAs. The necessary facilities for detecting the emission of evolved gases from the combustion of reprocessed PLAs could thus be the same than those 
J.D. Badia, L. Santonja-Blasco, A. Martínez-Felipe,A. Ribes-Greus. Reprocessed polylactide: studies of thermooxidative decomposition. Bioresource Technology 2012; 114:622-628.

needed for virgin PLA, which may reduce costs of investing and implementation in new technologies.

\section{Conclusions}

Multi-rate linear-non-isothermal thermogravimetric (TGA) experiments under oxidative $\left(\mathrm{O}_{2}\right)$ conditions, coupled to FT-IR analysis for gas detection, were suitable to simulate the thermal behavior of virgin and multiple-injected PLA facingcombustion.A kinetic methodology was applied accounting for the evolution of the activation parameters along the decomposition. A powered equation was used to explain the variations of activation energy and pre-exponential factor along the decomposition process.A nucleation and growth model which gave importance to the formation of gas bubbles in the polymer melt was valid for all materials. Reprocessed PLA did not modify the profile of evolved gases.

\section{Acknowledgements}

The authors would like to acknowledge the Spanish Ministry of Science and Innovation for the financial support through the Research ProjectsENE2007-67584-C03, UPOVCE-3E-013, ENE2011-28735-C02-01, IT-2009-0074, as well as for the pre-doctoral research position for L. Santonja-Blasco through the FPI program. The Spanish Ministry for Education is acknowledged for the concession of a pre-doctoral research position to J.D. Badia and A. Martinez-Felipe by means of the FPU program. The authors thank the financial support of the Generalitat Valenciana through the ACOMP/2011/189, the Grisolia research position for A. Martínez-Felipe, and for the Forteza technician position.Universitat Politècnica de València (UPV, Spain) is thanked for additional support through the PAID 05-09-4331 and PAID 06-11-2037 
J.D. Badia, L. Santonja-Blasco, A. Martínez-Felipe,A. Ribes-Greus. Reprocessed polylactide: studies of thermooxidative decomposition. Bioresource Technology 2012; 114:622-628.

projects.AIMPLAS (Technological Institute of Plastic) is acknowledged for providing and processing the material. 
J.D. Badia, L. Santonja-Blasco, A. Martínez-Felipe,A. Ribes-Greus. Reprocessed polylactide: studies of thermooxidative decomposition. Bioresource Technology 2012; 114:622-628.

\section{References}

(Akahira and Sunose, 1971) Akahira, T., Sunose, T., 1971. Res. Rep. Chiba Inst. Technol. 16,22-31.

(Al-Salem et al, 2009) Al-Salem, S. M., Lettieri, P., Baeyens, J., 2009. Recycling and recovery routes of plastic solid waste (PSW): A review. Waste management. 29, 26252643.

(Badia et al, 2009) Badia, J.D., Vilaplana, F., Karlsson, S., Ribes-Greus, A., 2009. Thermal analysis as a quality tool for assessing the influence of thermomechanical degradation on recycled poly(ethylene terephthalate). Polymer Testing. 28, 169-175.

(Badia et al, 2010) Badia, J.D., Santonja-Blasco, L., Moriana, R., Ribes-Greus, A., 2010.Thermal analysis applied to the characterization of degradation in soil of polylactide: II. On the thermal stability and thermal decomposition kinetics.Polymer Degradation and Stability. 95, 2192-2199.

(Badia et al, 2011a) Badia, J.D., Strömberg, E., Ribes-Greus, A., Karlsson, S., 2011. A statistical design of experiments for optimizing the MALDI-TOF-MS sample preparation of polymers.An application in the assessment of the thermo-mechanical degradation mechanisms of poly (ethylene terephthalate).AnalyticaChimicaActa. 692, $85-95$.

(Badia et al, 2011b) Badia, J.D., Strömberg, E., Ribes-Greus, A., Karlsson, S., 2011.Assessing the MALDI-TOF MS sample preparation procedure to analyze the influence of thermo-oxidative ageing and thermo-mechanical degradation on poly (lactide). EuropeanPolymerJournal. 47, 1416-1428. 
J.D. Badia, L. Santonja-Blasco, A. Martínez-Felipe,A. Ribes-Greus. Reprocessed polylactide: studies of thermooxidative decomposition. Bioresource Technology 2012; 114:622-628.

.(Badia et al, 2012a) Badia, J.D., Strömberg, E.,Karlsson, S.,Ribes-Greus, A., 2012.The role of crystalline, mobile amorphous and rigid amorphous fractions in the performance of recycled poly (ethylene terephthalate) (PET).Polymer Degradation and Stability.97, 98-107.

(Badia et al, 2012b) Badia, J.D., Strömberg, E.,Karlsson, S.,Ribes-Greus, A., 2012.Material valorisation of amorphous polylactide. Influence of thermo-mechanical degradation on the morphology, segmental dynamics, thermal and mechanical perfomance.Polymer Degradation and Stability.97, 670-678.

(Badia et al, 2012c) Badia, J.D., Santonja-Blasco, L., Martínez-Felipe, A., Ribes-Greus, A., 2012. Studies on combined material and energetic valorizations of bio-based polymers for the packaging industry: Pyrolysis of reprocessed polylactide.

BioresourceTechnology. In press

(Barneto et al, 2010) Barneto, A.G., Carmona, J.A., Alfonso, J.E.M., Serrano, R.S., 2010. Simulation of the thermogravimetry analysis of three non-wood pulps.Bioresource Technology. 101, 3220-3229.

(Bikiaris and Karayannidis, 1999) Bikiaris, D. N., Karayannidis, G., 1999.Effect of carboxylic end groups on thermooxidative stability of PET and PBT.Polymer Degradation and Stability. 66, 213-218.

(Bourbigot and Fontaine, 2010) Bourbigot, S., Fontaine, G., 2010. Flame retardancy of polylactide: an overview. Polymer chemistry.1,1413-1422.

(Carrasco et al, 2010) Carrasco, F., Pagès, P., Gámez-Pérez, J., Santana, O.O., Maspoch, M.L., 2010.Kinetics of the thermal decomposition of processed poly(lactic acid). Polymer Degradation and Stability. 95, 2508-2514. 
J.D. Badia, L. Santonja-Blasco, A. Martínez-Felipe,A. Ribes-Greus. Reprocessed polylactide: studies of thermooxidative decomposition. Bioresource Technology 2012; 114:622-628.

(Coats and Redfern, 1964) Coats, A. W., Redfern, J. P., 1964.Kinetic analysis from thermogravimetric data.Nature.68, 4914.

(Doyle, 1965) Doyle, C. D., 1965.Series approximations to the equation of thermogravimetric data.Nature.207, 209.

(Flynn and Wall, 1966) Flynn, J. H., Wall, L. A., 1966.A quick, direct method for the determination of activation energy from thermogravimetric data.Journal of Polymer Science. 4, 323-342.

(Freundlich, 1928) Freundlich, H., 1928.Colloid and Capillary Chemistry.New York .Duton\& Co.

(Galwey, 2003) Galwey A.K., 2003. What is meant by the term 'variable activation energy' when applied in the kinetic analyses of solid state decompositions (crystolysis reactions)?.Thermochimica Acta, 397, 249-268,

(Gotor et al, 2000) Gotor, F.J., Criado, J.M., Malek,J., Koga, K., 2000. Kinetic analysis of solid-state reactions: The universality of Master Plots for analyzing isothermal and non-isothermal experiments. Journal of Physical Chemistry A. 104, 10777-10782.

(Gupta and Kumar, 2007) Gupta, A. P., Kumar, V., 2007. New emerging trends in synthetic biodegradable polymers - Polylactide: a critique. European Polymer Journal 43, 4053-4074.

(Khawan and Flanagan, 2006) Khawan, A., Flanagan, D. R., 2006. Solid-State Kinetic Models: Basics and Mathematical Fundamentals.Journal of Physical Chemistry B. 110, 17315-17328. 
J.D. Badia, L. Santonja-Blasco, A. Martínez-Felipe,A. Ribes-Greus. Reprocessed polylactide: studies of thermooxidative decomposition. Bioresource Technology 2012; 114:622-628.

(Kissinger, 1957) Kissinger, H. E., 1957.Reaction kinetics in differential thermal analysis.Analytical Chemistry. 29, 1702-1706.

(Liu et al, 2006) Liu,X.,Zou,Y.,Li,W.,Cao,G.,Chen,W., 2006. Kinetics of thermooxidative and thermal degradation of poly(D,L- Lactide) (PDLLA) at processing temperature . Polymer Degradation and Stability. 91, 3259-3265.

(Materazzi and Vecchio, 2010)Materazzi, S., Vecchio, S., 2010.Evolved Gas Analysis by Infrared Spectroscopy.Applied Spectroscopy Reviews. 45, 241-273

(Morita, 2005) Morita, S. 2D Shige (c). - Kwansei-Gakuin University- .2004-2005.

(Noda, 1990) Noda, I., 1990. Two-Dimensional Infrared (2D IR) Spectroscopy: Theory and Applications. Applied Spectroscopy.44, 550-561.

(Ozawa, 1970) Ozawa, T., 1970. Kinetic analysis of derivative curves in thermal analysis. Journal of ThermalAnalysis.2, 301.

(Pérez-Maqueda et al, 2002) Pérez-Maqueda, L. A., Criado, J. M., Gotor, F. J., Málek, J., 2002. Advantages of combined kinetic analysis of experimental data obtained under any heating profile.Journal of PhysicalChemistry A. 106, 2862-2868.

(Strömberg and Karlsson, 2009)Strömberg, E., Karlsson, S., 2009.The design of a test protocol to model the degradation of polyolefins during recycling and service life.Journal of Applied Polymer Science. 112, 1835-1844.

(Vilaplana and Karlsson, 2008)Vilaplana, F., Karlsson, S., 2008.Quality concepts for the improved use of recycled polymeric materials: a review.Macromolecular Materials and Engineering.293, 274-297. 
J.D. Badia, L. Santonja-Blasco, A. Martínez-Felipe,A. Ribes-Greus. Reprocessed polylactide: studies of thermooxidative decomposition. Bioresource Technology 2012; 114:622-628.

(Vogel and Siesler, 2008) Vogel,C., Siesler, H. W., 2008. Thermal degradation of poly(ع-caprolactone), poly(L-lactic acid) and their blends with poly(3-hydroxy-butyrate) studied by TGA/FT-IR spectroscopy. Macromolecular Symposia.265, 183-194.

(Vyazovkin, 1997) Vyazovkin, S., 1997.Advanced Isoconversional Method.Journal of Thermal Analysis. 49, 1493-1499.

(Vyazovkin, 2003) Vyazovkin, S., 2003. Reply to "What is meant by the term "variable activation energy' when applied in the kinetics analyses of solid state decompositions (crystolysis reactions)?’.ThermochimicaActa. 397, 269-271.

(Vyazovkin et al, 2011) Vyazovkin, S.,Burnham, A. K., Criado, J. M., Pérez-Maqueda, L.A.,Popescu, C., Sbirrazzuoli, N., 2011.ICTAC Kinetics Committee recommendations for performing kinetic computations on thermal analysis data, ThermochimicaActa, $520,1-19$. 
J.D. Badia, L. Santonja-Blasco, A. Martínez-Felipe,A. Ribes-Greus. Reprocessed polylactide: studies of thermooxidative decomposition. Bioresource Technology 2012; 114:622-628.

\section{ANNEX. OPEN-ACCESS POLICIES}

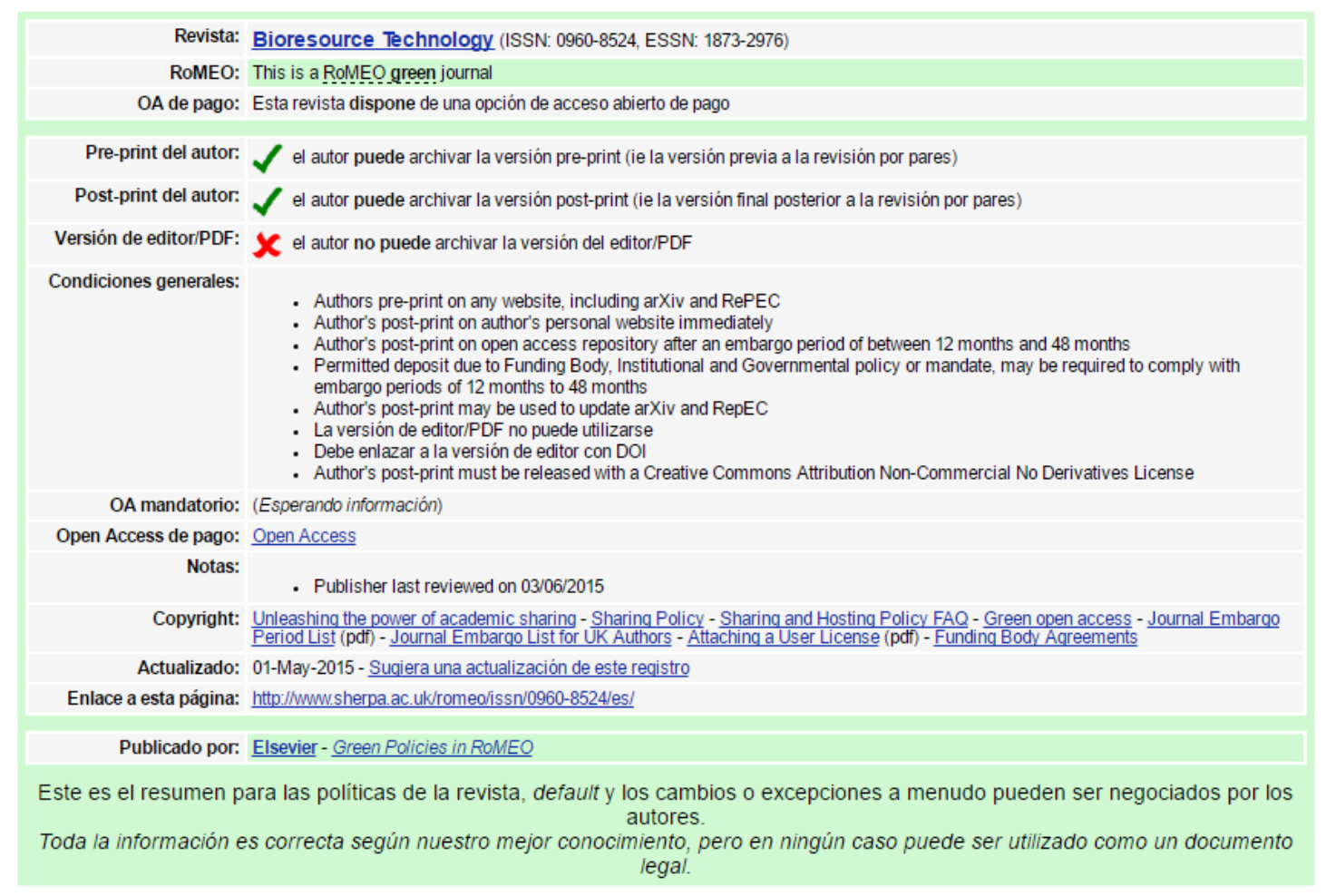

